\title{
A REMARK ON THE DERIVATIVE OF THE ONE-DIMENSIONAL HARDY-LITTLEWOOD MAXIMAL FUNCTION
}

\author{
Hitoshi TANAKA
}

Dedicated to Professor Kôzô Yabuta on the occasion of his 60th birthday

J. Kinnunen proved that if $p>1, d \leqslant 1$ and $f$ is a function in the Sobolev space $W^{1, p}\left(\mathbf{R}^{d}\right)$, then the first order weak partial derivatives of the Hardy-Littlewood maximal function $\mathcal{M} f$ belong to $L^{p}\left(\mathbf{R}^{d}\right)$. We shall show that, when $d=1$, Kinnunen's result can be extended to the case where $p=1$.

\section{RESUlt}

The derivative of the maximal function has been studied in, for example, Kinnunen [3], Kinnunen and Lindqvist [4] and Buckley [1].

For a locally integrable function $f$ on $\mathbf{R}^{d}$, where $d \geqslant 1$, the Hardy-Littlewood maximal function $\mathcal{M} f$ is defined by

$$
\mathcal{M} f(x)=\sup _{Q \ni x} \frac{1}{|Q|} \int_{Q}|f(y)| d y
$$

where the supremum is taken over all cubes $Q$ containing $x \in \mathbf{R}^{d}$. Here, $|Q|$ denotes the volume of the cube $Q$. The well-known theorem of Hardy, Littlewood and Wiener asserts the following. If $f \in L^{p}\left(\mathbf{R}^{d}\right)$, where $1<p \leqslant \infty$, then $\mathcal{M} f \in L^{p}\left(\mathbf{R}^{d}\right)$ and

$$
\|\mathcal{M} f\|_{p} \leqslant A_{p}\|f\|_{p}
$$

where the constant $A_{p}$ depends only on $p$ and the dimension $d$. If $f \in L^{1}\left(\mathbf{R}^{d}\right)$, then for every $\lambda>0$

$$
\left|\left\{x \in \mathrm{R}^{d}: \mathcal{M} f(x)>\lambda\right\}\right| \leqslant \frac{A}{\lambda}\|f\|_{1},
$$

where the constant $A$ depends only on $d$. Recall that when $1 \leqslant p \leqslant \infty$, the Sobolev space $W^{1, p}\left(\mathbf{R}^{d}\right)$ consists of functions $f$ in $L^{p}\left(\mathbf{R}^{d}\right)$ whose first order weak partial derivatives $D_{i} f$ belong to $L^{p}\left(\mathbf{R}^{d}\right)$, when $i=1,2, \ldots, d$.

\footnotetext{
Received 14th August, 2001

Supported by Japan Society for the Promotion of Sciences and Fujukai Foundation.

The author wishes to express his sincere thanks to S.T. Kuroda and Takeshi Hatakeyama for helpful discussions, which streamlined the original proof.
}

Copyright Clearance Centre, Inc. Serial-fee code: 0004-9727/02 \$A2.00+0.00. 
In $[3]$, Kinnunen showed that if $f \in W^{1, p}\left(\mathbf{R}^{d}\right)$, where $1<p<\infty$ and $d \geqslant 1$, then $\mathcal{M} f \in W^{1, p}\left(\mathbf{R}^{d}\right)$ and

$$
\left|\left(D_{i} \mathcal{M} f\right)(x)\right| \leqslant\left(\mathcal{M} D_{i} f\right)(x), \quad i=1,2, \ldots, d,
$$

for almost every $x \in \mathbf{R}^{d}$. Equations (2) and (3) imply that

$$
\left\|D_{i} \mathcal{M} f\right\|_{p} \leqslant A_{p}\left\|D_{i} f\right\|_{p} \quad i=1,2, \ldots, d .
$$

Kinnunen's method to prove (3) cannot be applied to the case where $p=1$, since it depends on the $L^{p}$-boundedness of $\mathcal{M}$.

The purpose of this paper is to extend (4) to the case where $p=d=1$. Notice that if $f \in W^{1,1}(\mathbf{R})$, then $\mathcal{M} f$ is a bounded function and hence is differentiable in the sense of distributions.

THEOREM 1. If $f \in W^{1,1}(\mathbf{R})$, then the derivative of $\mathcal{M} f$ is an integrable function, and

$$
\left\|(\mathcal{M} f)^{\prime}\right\|_{1} \leqslant 2\left\|f^{\prime}\right\|_{1} .
$$

Kinnunen proved his results for the maximal function which is defined as the supremum taken over all balls centred at $x$. If one reads [3] carefully, then one sees that the corresponding results hold for the maximal function which is defined as (1).

\section{ProOF}

A crucial point in our argument is to consider one-sided maximal functions. For a locally integrable function $f$ on the line, define the one-sided maximal functions $\mathcal{M}_{l} f$ and $\mathcal{M}_{r} f$ by

$$
\begin{aligned}
& \mathcal{M}_{l} f(x)=\sup _{s>0} \frac{1}{s} \int_{x-s}^{x}|f(y)| d y, \\
& \mathcal{M}_{r} f(x)=\sup _{t>0} \frac{1}{t} \int_{x}^{x+t}|f(y)| d y .
\end{aligned}
$$

The following relation is obvious,

$$
\mathcal{M} f(x)=\max \left\{\mathcal{M}_{l} f(x), \mathcal{M}_{r} f(x)\right\} .
$$

In the rest of this paper, we assume that $f \in W^{1,1}(\mathbf{R})$, and we shall state the results only for $\mathcal{M}_{l}$, but the corresponding results hold for $\mathcal{M}_{r}$ as well. Notice that if $f \in W^{1,1}(\mathbf{R})$, then (after adjusting on a set of measure zero) $f$ may be taken to be continuous - and then $f$ vanishes at infinity, for it is uniformly continuous and integrable. Notice further that then $\mathcal{M}_{l} f$ is continuous and vanishes at infinity (see the proof of Theorem 4.1 in [3]). Therefore, the set

$$
E=\left\{x \in \mathbf{R}: \mathcal{M}_{l} f(x)>|f(x)|\right\}
$$


is open and hence $E$ can be written as

$$
E=\bigcup_{j} I_{j}=\bigcup_{j}\left(\alpha_{j}, \beta_{j}\right)
$$

where $\left(\alpha_{j}, \beta_{j}\right)$ are disjoint open intervals.

LEMMA 2. With the definitions above, the following hold.

(a) $\mathcal{M}_{l} f$ is a nonincreasing function on each $I_{j}$.

(b) $\mathcal{M}_{l} f$ is a locally Lipschitz function on each $I_{j}$. In particular, $\mathcal{M}_{l} f$ is an absolutely continuous function on each compact subinterval of $I_{j}$.

Proof: (a) Take $K=[\alpha, \beta] \subset I_{j}$. It suffices to prove that $\mathcal{M}_{l} f$ is nonincreasing on $K$. By the continuity of $|f|$ and $\mathcal{M}_{l} f$ we have

$$
\varepsilon \equiv \inf _{x \in K} \mathcal{M}_{l} f(x)-|f(x)|>0 .
$$

By the uniform continuity of $|f|$ there exists $\delta>0$ such that

$$
|f(y)|<|f(x)|+\frac{\varepsilon}{2}, \quad x \in K,|y-x| \leqslant \delta .
$$

The definition of $\varepsilon$ and (6) imply that

$$
\mathcal{M}_{l} f(x)=\sup _{s>\delta} \frac{1}{s} \int_{x-s}^{x}|f(y)| d y, \quad x \in K .
$$

We shall see that

$$
\mathcal{M}_{l} f(x-h) \geqslant \mathcal{M}_{l} f(x), \quad x-h, x \in K, 0<h \leqslant \delta .
$$

Suppose that $s>\delta$. Then, from (6),

$$
\begin{aligned}
\frac{1}{s} \int_{x-s}^{x}|f(y)| d y & =\frac{s-h}{s} \cdot \frac{1}{s-h} \int_{x-s}^{x-h}|f(y)| d y+\frac{h}{s} \cdot \frac{1}{h} \int_{x-h}^{x}|f(y)| d y \\
& \leqslant \max \left\{\mathcal{M}_{l} f(x-h),|f(x)|+\frac{\varepsilon}{2}\right\} .
\end{aligned}
$$

Taking the supremum on the left-hand side of $(9)$ when $s>\delta$, we have

$$
\mathcal{M}_{l} f(x) \leqslant \max \left\{\mathcal{M}_{l} f(x-h),|f(x)|+\frac{\varepsilon}{2}\right\}
$$

by (7). By the definition of $\varepsilon$ we also have $\mathcal{M}_{l} f(x) \geqslant|f(x)|+\varepsilon$. Thus, we obtain (8).

(b) Let $K$ and $\delta$ be as in the proof of (a). Suppose that $x, x+h \in K, h>0$, and $s>\delta$. Then it follows from (a) that 


$$
\begin{aligned}
\frac{1}{s} \int_{x-s}^{x}|f(y)| d y-\mathcal{M}_{l} f(x+h) & \leqslant \frac{1}{s} \int_{x-s}^{x}|f(y)| d y-\frac{1}{s+h} \int_{x-s}^{x+h}|f(y)| d y \\
& \leqslant \frac{1}{s} \int_{x-s}^{x}|f(y)| d y-\frac{1}{s+h} \int_{x-s}^{x}|f(y)| d y \\
& =\frac{1}{s+h} \cdot \frac{1}{s} \int_{x-s}^{x}|f(y)| d y \cdot h \\
& \leqslant \frac{\mathcal{M}_{l} f(x)}{\delta} \cdot h \\
& \leqslant \frac{\mathcal{M}_{l} f(\alpha)}{\delta} \cdot h .
\end{aligned}
$$

Taking the supremum on the left-hand side of $(10)$ when $s>\delta$, we obtain

$$
0 \leqslant \mathcal{M}_{l} f(x)-\mathcal{M}_{l} f(x+h) \leqslant C h
$$

by (7) and (a).

Proposition 3. If $f \in W^{1,1}(\mathbf{R})$, then the distributional derivatives of $\mathcal{M}_{l} f$ and $\mathcal{M}_{r} f$ are integrable functions, and

$$
\left\|\left(\mathcal{M}_{l} f\right)^{\prime}\right\|_{1} \leqslant\left\|f^{\prime}\right\|_{1}, \quad\left\|\left(\mathcal{M}_{r} f\right)^{\prime}\right\|_{1} \leqslant\left\|f^{\prime}\right\|_{1} .
$$

Proof: We shall prove the proposition only for $\mathcal{M}_{l} f$. We note that if $f \in W^{1,1}(\mathbf{R})$, then $|f| \in W^{1,1}(\mathbf{R})$ and

$$
\left\||f|^{\prime}\right\|_{1}=\left\|f^{\prime}\right\|_{1}
$$

(see [2]).

Recall that

$$
E=\bigcup_{j} I_{j}=\bigcup_{j}\left(\alpha_{j}, \beta_{j}\right) .
$$

Set $F=\mathbf{R} \backslash E$. From Lemma $2, \mathcal{M}_{l} f$ is diferentiable almost everywhere on each $I_{j}$, and the derivative, $v$ say, satisfies $v \leqslant 0$. We shall prove that the weak derivative of $\mathcal{M}_{l} f$ is given by

$$
\left(\mathcal{M}_{l} f\right)^{\prime}=\chi_{E} v+\chi_{F}|f|^{\prime},
$$

where $\chi_{E}$ and $\chi_{F}$ denote the indicator functions of the sets $E$ and $F$.

For a test function $\phi \in \mathcal{D}(\mathbf{R})$ we see that

$$
\int_{I_{j}} \mathcal{M}_{l} f(y) \phi^{\prime}(y) d y=\left[\left|f\left(\beta_{j}\right)\right| \phi\left(\beta_{j}\right)-\left|f\left(\alpha_{j}\right)\right| \phi\left(\alpha_{j}\right)\right]-\int_{I_{j}} v(y) \phi(y) d y
$$

by the continuity of $\mathcal{M}_{l} f$ and a limiting argument. (Here, and later, if $\alpha_{j}=-\infty$ or if $\beta_{j}=+\infty$, then $f\left(\alpha_{j}\right)=0$ and $f\left(\beta_{j}\right)=0$; similar remarks apply to $\mathcal{M}_{l} f\left(\alpha_{j}\right)$ and 
$\left.\mathcal{M}_{l} f\left(\beta_{j}\right).\right)$ It follows from (14) that

$$
\begin{aligned}
\int_{\mathbf{R}} \mathcal{M}_{l} f(y) \phi^{\prime}(y) d y \\
\quad=\int_{E \cup F} \mathcal{M}_{l} f(y) \phi^{\prime}(y) d y \\
=\sum_{j}\left[\left|f\left(\beta_{j}\right)\right| \phi\left(\beta_{j}\right)-\left|f\left(\alpha_{j}\right)\right| \phi\left(\alpha_{j}\right)\right]-\int_{E} v(y) \phi(y) d y+\int_{F}|f(y)| \phi^{\prime}(y) d y \\
=\int_{E}|f(y)| \phi^{\prime}(y) d y+\int_{E}|f|^{\prime}(y) \phi(y) d y-\int_{E} v(y) \phi(y) d y+\int_{F}|f(y)| \phi^{\prime}(y) d y \\
=\int_{\mathbf{R}}|f(y)| \phi^{\prime}(y) d y+\int_{E}|f|^{\prime}(y) \phi(y) d y-\int_{E} v(y) \phi(y) d y \\
=-\int_{\mathbf{R}}\left(\chi_{E}(y) v(y)+\chi_{F}(y)|f|^{\prime}(y)\right) \phi(y) d y .
\end{aligned}
$$

This relation implies (13).

Now, we shall prove (11). For each interval $I_{j}$, since $v \leqslant 0$, we have

$$
\begin{aligned}
\int_{I_{j}}|v(y)| d y & =\mathcal{M}_{l} f\left(\alpha_{j}\right)-\mathcal{M}_{l} f\left(\beta_{j}\right) \\
& =\left|f\left(\alpha_{j}\right)\right|-\left|f\left(\beta_{j}\right)\right| \\
& =-\int_{I_{j}}|f|^{\prime}(y) d y \leqslant\left.\int_{I_{j}}|| f\right|^{\prime}(y) \mid d y .
\end{aligned}
$$

From (15) and (12) we obtain

$$
\left\|\left(\mathcal{M}_{l} f\right)^{\prime}\right\|_{1}=\int_{E}|v|+\left.\int_{F}|| f\right|^{\prime}\left|\leqslant\left\||f|^{\prime}\right\|_{1}=\left\|f^{\prime}\right\|_{1} .\right.
$$

We need one more lemma.

Lemma 4. Let $f$ and $g$ be (real valued) integrable functions on the line, and set $F(x)=\int_{-\infty}^{x} f(y) d y, G(x)=\int_{-\infty}^{x} g(y) d y$, and $H(x)=\max \{F(x), G(x)\}$. Then the weak derivative of $H$ is an integrable function, and

$$
\left\|H^{\prime}\right\|_{1} \leqslant\|f\|_{1}+\|g\|_{1} .
$$

This lemma can be proved easily (see [2, Lemma 7.6]).

The theorem now follows from (5), Lemma 4 and Proposition 3.

\section{REFERENCES}

[1] S. Buckley, 'Is the maximal function of a Lipschitz function continuous?', Ann. Acad. Sci. Fenn. Math. 24 (1999), 519-528.

[2] D. Gilbarg and N.S. Trudinger, Elliptic partial differential equations of second order, Grundlehren der Mathematischen Wissenschaften 224, (2nd edition) (Springer-Verlag, Berlin, Heidelberg, New York, 1983). 
[3] J. Kinnunen, 'The Hardy-Littlewood maximal function of a Sobolev function', Israel J: Math. 100 (1997), 117-124.

[4] J. Kinnunen and P. Lindqvist, 'The derivative of the maximal function,', J. Reine Angew. Math. 503 (1998), 161-167.

Department of Mathematics

Gakushuin University

1-5-1 Mejiro, Toshima-ku

Tokyo $171-8588$

Japan

e-mail: hitoshi.tanaka@gakushuin.ac.jp 